\title{
MicroRNA-433 regulates apoptosis by targeting PDCD4 in human osteosarcoma cells
}

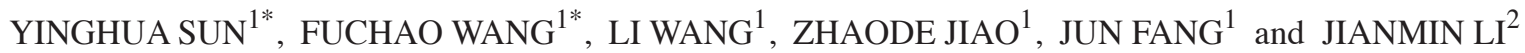 \\ ${ }^{1}$ Department of Orthopedics, Yidu Central Hospital, Weifang Medical University, Weifang, Shandong 262500; \\ ${ }^{2}$ Department of Orthopedics, Qilu Hospital, Shandong University, Jinan, Shandong 250012, P.R. China
}

Received May 28, 2015; Accepted July 16, 2016

DOI: $10.3892 / 01.2017 .6441$

\begin{abstract}
Osteosarcoma is the most common aggressive sarcoma of the bone in children and adolescents. It is characterized by a high level of genetic instability and recurrent DNA deletions and amplifications. microRNAs (miRNAs) play a key role in cancer initiation, progression and metastasis; however, the potential role of miRNAs in osteosarcoma remains largely unknown. In the present study, miR-433 was shown to be overexpressed in osteosarcoma tissues compared with normal human osteoblasts. Transfection of miR-433 mimics into osteosarcoma cell lines significantly decreased apoptosis by targeting programmed cell death 4, a tumor suppressor that is involved in apoptosis. In contrast, inhibition of miR-433 enhanced apoptosis. Furthermore, in vivo miR-433 overexpression inhibited the apoptosis of tumor cells and increased tumor growth. The results of the present study suggested that miR-433 is a potential molecular target for osteosarcoma therapy.
\end{abstract}

\section{Introduction}

Osteosarcoma is the most common aggressive bone tumor in children and adolescents (1). Although many tumors initially respond to chemotherapy, patients with metastatic or relapsed osteosarcoma have extremely poor survival outcomes (2). The 5 -year survival rate of patients without metastatic disease is $60-70 \%$, while the clinical outcomes for patients with metastatic disease are far worse, since a 5-year survival rate of $15-30 \%$ has been reported for these patients $(3,4)$. However, the mechanisms underlying the multiple oncogenic properties of osteosarcoma are not fully understood. A comprehensive

Correspondence to: Dr Jianmin Li, Department of Orthopedics, Qilu Hospital, Shandong University, 107 West Wenhua Road, Jinan, Shandong 250012, P.R. China

E-mail: jianmin365@126.com

*Contributed equally

Key words: miR-433, programmed cell death 4, apoptosis, osteosarcoma understanding of the mechanisms is required for the development of effective therapeutic strategies.

Previous studies have revealed that microRNAs (miRNAs) regulate numerous cellular processes, including those associated with cancer (5). miRNAs are a class of small non-coding RNAs that range from 22-25 nucleotides in length (6). The 5 '-region of a miRNA contains 2-8 bases, termed the seed region, which is important for target $\mathrm{mRNA}$ recognition (7). miRNAs negatively regulate gene expression by inhibiting protein translation or by causing mRNA degradation through partial or complete base-pairing with the $3^{\prime}$ untranslated region (3'UTR) of the target mRNA. Dysregulation of miRNA expression has been reported in various human cancers $(5,7)$. Therefore, exploring the mechanisms underlying the carcinogenic effects of these aberrant tumor miRNAs is critical for cancer diagnosis and therapy.

Dysregulated miR-433 expression has been observed in various cancers and has been significantly associated with the clinical outcome of tumor patients. For example, the overexpression of miR-433 in a gastric cancer cell line suppressed cell growth and invasion by downregulating KRAS expression (8). Overexpression of miR-433 downregulated thymidylate synthetase mRNA and protein expression and enhanced the sensitivity of HeLa cells to 5-fluorouracil (9). In a previous study, miR-433 expression was decreased in myeloproliferative neoplasms, and overexpression of miR-433 was shown to inhibit hematopoietic cell growth and differentiation by targeting guanylate binding protein 2 (GBP2) (10). However, the role of miR-433 in osteosarcoma cells remains unknown.

In the present study, miR-433 was shown to be upregulated in human osteosarcoma tissue compared with normal tissue. Ectopic expression of miR-433 decreased apoptosis in osteosarcoma cells by targeting programmed cell death 4 (PDCD4), indicating that miR-433 may be a potential molecular target for osteosarcoma treatment.

\section{Materials and methods}

Cell culture. Two human osteosarcoma cell lines, U2OS and G293, were obtained from American Type Culture Collection (Manassas, VA, USA). The normal human osteoblastic cell line hFOB 1.19 (American Type Culture Collection, Manassas, VA, USA) was used as a control. The cells were cultured in Dulbecco's modified Eagle's medium supplemented with $10 \%$ 
heat-inactivated fetal calf serum (HyClone; GE Healthcare Life Sciences, Logan, UT, USA) at $37^{\circ} \mathrm{C}$ with $5 \% \mathrm{CO}_{2}$ and $95 \%$ humidity.

Patients. Three paired osteosarcoma tissues and normal adjacent tissues were obtained from each patient by biopsy prior to any treatment. Histological diagnosis demonstrated that all tumor samples were high-grade osteosarcoma with either stage IIA or IIB in the Enneking surgical staging system.

RNA extraction and reverse transcription-quantitative polymerase chain reaction ( $q P C R$ ). Total RNA was extracted from clinical specimens and cell lines using TRIzol reagent (Thermo Fisher Scientific, Inc., Waltham, MA, USA), according to the manufacturer's protocol. Reverse transcription was performed using the TaqMan MicroRNA Reverse Transcription kit (Applied Biosystems; Thermo Fisher Scientific, Inc.). qPCR was performed using SYBR Green Real-Time PCR Master Mix (Roche Diagnostics, Basel, Switzerland) on the Applied Biosystems 7500 Real-Time PCR System (Thermo Fisher Scientific, Inc.). The cycling conditions were as follows: $94^{\circ} \mathrm{C}$ for $5 \mathrm{~min}$, followed by 40 cycles of $94^{\circ} \mathrm{C}$ for $10 \mathrm{sec}, 60^{\circ} \mathrm{C}$ for $45 \mathrm{sec}$ and $72^{\circ} \mathrm{C}$ for $30 \mathrm{sec}$. The relative expression of miR-433 was normalized to U6 small nuclear RNA (RNU6) expression. Each experiment was performed in triplicate. All samples were normalized to the internal control, and fold-changes were calculated using the $2^{-\Delta \Delta \mathrm{Cq}}$ quantification method (11). The primers used for miR-433 and RNU6 (Sigma-Aldrich; Merck Millipore, Darmstadt, Germany) were as follows: miR-433, forward 5'-GGCGGTGAATAATGAC-3' and reverse 5'-GTG CAGGGTCCGAGGT-3'; and RNU6, forward 5'-GTCGTA TCCAGTGCAGGGTCCGAGGTATTCGCACTGGATACG ACTAAGCA-3' and reverse 5'-CCTGCGCAAGGATGAC-3'.

Microarray data analysis. Expression data (Gene Expression Omnibus accession no. 39040; title: MicroRNA profiling and clinical outcomes in human osteosarcoma) were downloaded and imported to the $\mathrm{R}$ environment available at http://www.r-project.org and processed using the Illumina-specific package lumi. Variance-stabilizing transformation (12) and quantile normalization were applied to analyze gene expression.

Transfections. Synthetic constructs containing miR-433 mimic, miR-433 inhibitor, negative control or PDCD4 small interfering (si)RNA (GenePharma Co., Ltd., Shanghai, China) were transfected into the osteosarcoma cell lines at a final concentration of $20 \mathrm{nM}$. All transfections were performed using Lipofectamine 2000 (Invitrogen; Thermo Fisher Scientific, Inc.), according to the manufacturer's protocol.

Vector construction. PDCD4 was identified as a potential target for miR-433 using TargetScan (http:/www.targetscan.org/) and miRanda (http://www.microrna.org/). The fragment of the 3'UTR ofPDCD4mRNA(GenBankaccessionno.NM014456.4) containing the potential miR-433 binding site was amplified by PCR using forward 5'-TGCCATGTTTATTATCTAA-3' and reverse 5'-AATTCGAGATCCAGTCTA-3' primers, and cloned downstream of the luciferase reporter gene in the pGL3-control vector (Promega Corporation, Madison, WI,
USA) to form pGL3-PDCD4-3'UTR-WT. The 3'UTR-Mut plasmid of PDCD4 (pGL3-PDCD4-3'UTR-MUT) harboring mutations in the sequence complementary to the seed region of miR-433 was constructed using site-specific mutagenesis (13).

Luciferase reporter assay. The dual-luciferase reporter assay was performed to determine whether miR-433 downregulates PDCD4 by binding to the 3'UTR region. HEK293T cells (American Type Culture Collection) were plated in a 48-well plate at $80-90 \%$ confluence. After cotransfection with $20 \mathrm{nM}$ miR-433 mimic or scramble control and $40 \mathrm{ng}$ pGL3-PDCD4-3'UTR-WT or pGL3-PDCD4-3'UTR-MUT, the cells were collected to measure Firefly luciferase activity with the dual-luciferase assay (Promega Corporation). The assays were performed in triplicate.

Western blotting. The cells were lysed using radioimmunoprecipitation assay lysis buffer (Invitrogen; Thermo Fisher Scientific, Inc.) containing $1 \mathrm{X}$ phenylmethylsulfonyl fluoride and $1 \mathrm{X}$ protease inhibitor cocktail (Roche Diagnostics). The protein concentration was measured using the bicinchoninic acid protein assay kit (Pierce; Thermo Fisher Scientific, Inc.). The proteins $(30 \mu \mathrm{g})$ were separated by $10 \%$ SDS-PAGE and transferred onto a PVDF membrane. The membranes were blocked with $5 \%$ nonfat milk in TBST for $1 \mathrm{~h}$ and incubated overnight at $4^{\circ} \mathrm{C}$ with the following primary antibodies: Rabbit monoclonal anti- $\beta$-actin (1:5,000; catalog no. 4970; Cell Signaling Technology, Inc., Danvers, MA, USA); rabbit monoclonal anti-PDCD4 (1:1,000; catalog no. 9535; Cell Signaling Technology, Inc.); and rabbit monoclonal anti-PARP (1:1,000; catalog no. 9532; Cell Signaling Technology, Inc.). Upon being washed, the membranes were incubated with horseradish peroxidase-conjugated secondary antibodies (1:5,000; catalog no. ab97051; Abcam, Cambridge, UK) at room temperature for $1 \mathrm{~h}$. Signals were detected with an enhanced chemiluminescence system (Bio-Rad Laboratories, Inc., Hercules, CA, USA).

Terminal deoxynucleotidyl transferase dUTP nick end labeling (TUNEL) analysis. OCT-embedded cryosections (6 $\mu$ m-thick) of tumors were fixed with $4 \%$ paraformaldehyde for $30 \mathrm{~min}$. Following permeabilization with $0.1 \%$ Triton $\mathrm{X}-100$ for $10 \mathrm{~min}$, the tumor sections were incubated with methanol containing $3 \% \mathrm{H}_{2} \mathrm{O}_{2}$, followed by dUTP nick end labeling at $37^{\circ} \mathrm{C}$ for $1 \mathrm{~h}$ using the In Situ Cell Death Detection kit (Sigma-Aldrich; Merck Millipore).

Apoptosis analysis. Apoptosis was assessed used Annexin V/propidium iodide (PI) staining. Adherent and floating cells were harvested by trypsinization and washed three times with PBS. Subsequently, the cells were stained with $5 \mu \mathrm{l}$ Annexin $\mathrm{V}$ in $60 \mu \mathrm{l} 1 \mathrm{X}$ binding buffer (BD Biosciences, Franklin Lakes, NJ, USA) for $15 \mathrm{~min}$ at room temperature in the dark. After staining, $120 \mu \mathrm{l} 1 \mathrm{X}$ binding buffer and $5 \mu \mathrm{l} \mathrm{PI}$ were added to the cells, and the cells were analyzed using a FACSCalibur flow cytometer.

Immunofluorescence. Cells were fixed with $4 \%$ paraformaldehyde and treated with 1:400 diluted rabbit monoclonal anti-PDCD4 antibody (catalog no. 9535; Cell Signaling 
Technology, Inc.), followed by 1:400 diluted Alexa Fluor 568-conjugated anti-rabbit antibody (Cell Signaling Technology, Inc.) and DAPI (0.5 ng/ml; Thermo Fisher Scientific, Inc.).

Tumorigenicity assay in nude mice. A total of 10 4-to-6-week old male athymic nude mice, which weighed 24-30 g, were purchased from Shanghai Laboratory Animal Center (Shanghai, China). All mice were housed and treated in accordance with the guidelines of the Shandong University Animal Care and Use committee (Shanghai, China), and were maintained in specific pathogen-free conditions, consisting of a reversed 12:12-h light-dark cycle with controlled temperature $\left(21 \pm 1^{\circ} \mathrm{C}\right)$ and humidity. The mice were allowed free access to food and water. All experiments involving animals were undertaken in accordance with the Health Guide for the Care and Use of Laboratory Animals at Shandong University. U2OS cells $\left(1 \times 10^{6}\right.$ cells) stably expressing miR-433 or the control vector were inoculated bilaterally into the armpit region of 4-week-old immunodeficient nude mice. The tumor weights were measured 30 days following inoculation. All mice were euthanized by $30 \% \mathrm{CO}_{2}$ inhalation for $7 \mathrm{~min}$ and the tumor tissues were dissected.

Statistical analysis. Data are presented as the mean \pm standard deviation. Statistical analyses were performed using SPSS 17.0 software. Statistical significance was determined using the two-tailed unpaired Student's t-test, unless otherwise noted. $\mathrm{P}<0.05$ was considered to indicate a statistically significant difference.

\section{Results}

miR-433 is overexpressed in osteosarcoma. In order to detect the expression level of miR-433 in human osteosarcoma, the miRNA expression profile array (Gene Expression Omnibus accession no. 39040; title: MicroRNA profiling and clinical outcomes in human osteosarcoma) was analyzed and showed that miR-433 was significantly upregulated in osteosarcoma tissues compared with normal tissues ( $\mathrm{P}=0.0046$; Fig. 1A). To further confirm the upregulation of miR-433 in osteosarcoma, RT-qPCR analysis of three matched clinical sample pairs was performed. miR-433 expression was markedly higher in clinical osteosarcoma tissues compared with adjacent normal tissues (Fig. 1B). In addition, RT-qPCR was used to show that the miR-433 expression level in the normal human osteoblastic cell line hFOB 1.19 was lower compared with the human osteosarcoma cell lines (Fig. 1C).

miR-433 inhibits the apoptosis of human osteosarcoma cells in vitro. To assess the effects of miR-433 expression on osteosarcoma cell survival, the osteosarcoma cell line U2OS was used. Cells were transfected with miR-433 mimic, miR-433 inhibitor or scrambled control and were stained with Annexin V, a well known marker of early apoptosis. Flow cytometry showed that a significant decrease in Annexin V binding was detected at $48 \mathrm{~h}$ following transfection with miR-433 mimic compared with the control (Fig. 2A and B). As expected, the miR-433 inhibitor increased the apoptosis of U2OS cells compared with the control (Fig. 2A and B). To further confirm the effects of miR-433 on apoptosis in osteosarcoma cells, western blot analysis to assess the levels of cleaved poly(ADP-ribose) polymerase (PARP) was performed. The result showed that miR-433 mimics decreased the levels of cleaved PARP (Fig. 2C). These results suggest that overexpression of miR-433 protects the tumor cells from apoptosis.

miR-433 suppresses the expression of PDCD4. To further examine the mechanism by which miR-433 inhibits apoptosis in osteosarcoma cells, the present study analyzed the downstream targets of miR-433 using TargetScan and miRanda to predict potential direct targets $(14,15)$, in particular those involved in apoptosis. PDCD4, a tumor suppressor involved in apoptosis (16), had potential miR-433-binding regions in the 3'UTR of its mRNA (Fig. 3A). RT-qPCR and western blotting confirmed that miR-433 downregulated PDCD4 in osteosarcoma cells at both the protein and mRNA expression levels (Fig. 3B and C), which suggested that miR-433 regulates the expression of PDCD4 by promoting the degradation of its mRNA. To explore whether PDCD4 is indeed the target of miR-433, the luciferase reporter assay using vectors encoding wild-type or mutant PDCD4 3'UTR downstream of the luciferase gene was performed. Luciferase activity was markedly decreased in miR-433-overexpressing cells co-transfected with pGL3-PDCD4-3'UTR-WT, but not in cells co-transfected with pGL3-PDCD4-3'UTR-MUT (Fig. 3D).

miR-433 decreases tumor apoptosis in vivo. To examine the impact of miR-433 on tumor apoptosis, Balb/c nude mice were used to establish a xenograft tumor model. U2OS cells stably overexpressing miR-433 or vehicle were subcutaneously injected into the bilateral armpits of nude mice. Tumor weight was measured after sacrifice on day 30 post-injection. As shown in Fig. 4A and B, the tumor weight of the miR-433 overexpression group was significantly increased compared with the control group $(\mathrm{P}=0.0073)$. In situ TUNEL analysis showed that tumors overexpressing miR-433 had less TUNEL-positive cells than control group tumors (Fig. 4C). Immunofluorescence analysis of the xenograft tumor showed that tumors overexpressing miR-433 had decreased PDCD4 expression (Fig. 4D), which was consistent with the in vitro data, suggesting that miR-433 regulates apoptosis by targeting PDCD4.

\section{Discussion}

Osteosarcoma is the most common primary malignant tumor of the bone; it has an annual worldwide incidence of 1-3 cases per 1 million (17). Osteosarcoma occurs most frequently in children and adolescents, with a second peak in incidence in those aged $>50$ years (18). Despite significant improvements in the diagnosis and treatment of osteosarcoma, overall survival has remained relatively constant for $>20$ years (2). Several dysregulated miRNAs, including downregulated miR-34 and miR-143, and upregulated miR-140 and miR-21, have been associated with osteosarcoma development (19-21). However, the identification of novel and important dysregulated miRNAs, as well as the elucidation of their roles in osteosarcoma carcinogenesis and progression, remains an ongoing process.

Increasingly, studies have shown that various miRNAs involved in apoptosis are also associated with chemotherapy 
A

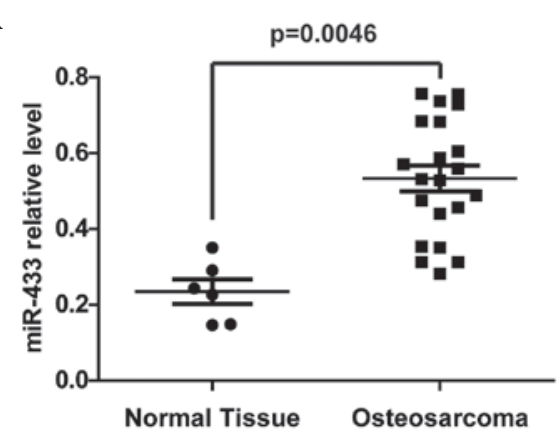

C

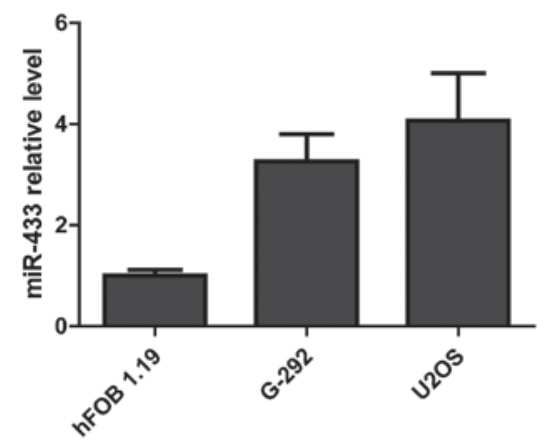

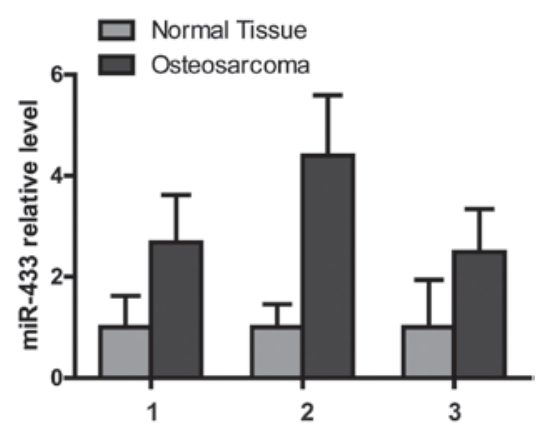

Figure 1. miR-433 is overexpressed in osteosarcoma tissues and cell lines. (A) miR-433 was significantly overexpressed in osteosarcoma tissues compared with normal tissues in a microRNA expression array. (B) RT-qPCR analysis showed that miR-433 expression was markedly higher in osteosarcoma tissues compared with matched normal tissues. (C) RT-qPCR analysis showed that miR-433 was overexpressed in osteosarcoma cell lines (G-292 and U2OS) compared with the normal human osteoblastic cell line hFOB 1.19. miR-433, microRNA-433; RT-qPCR, reverse transcription-quantitative polymerase chain reaction.

A

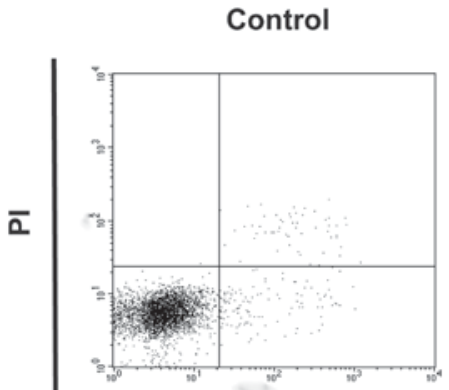

miR-433 mimics

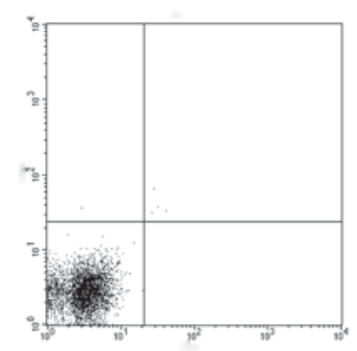

miR-433 inhibitor

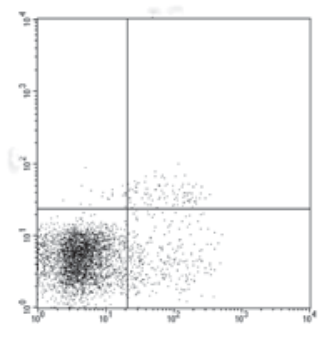

Annexin V

B

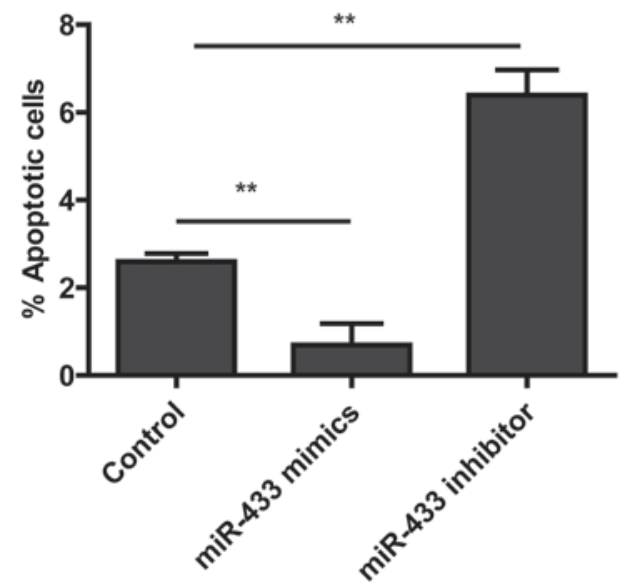

C

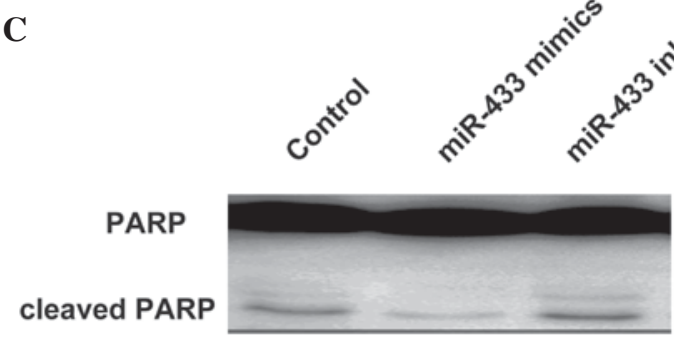

Figure 2. miR-433 promotes apoptosis of osteosarcoma cells. (A) U2OS cells were transfected with scramble, miR-433 mimic or miR-433 inhibitor, and then stained with PI and Annexin V and analyzed by flow cytometry. (B) Percentages of Annexin V-positive U2OS cells transfected with scramble, miR-433 mimic or miR-433 inhibitor. (C) Cleaved PARP n U2OS cells transfected with scramble, miR-433 mimic or miR-433 inhibitor was detected by western blotting. miR-433, microRNA-433; PI, propidium iodide; PARP, poly(ADP-ribose) polymerase.* $\mathrm{P}<0.05$. 
A

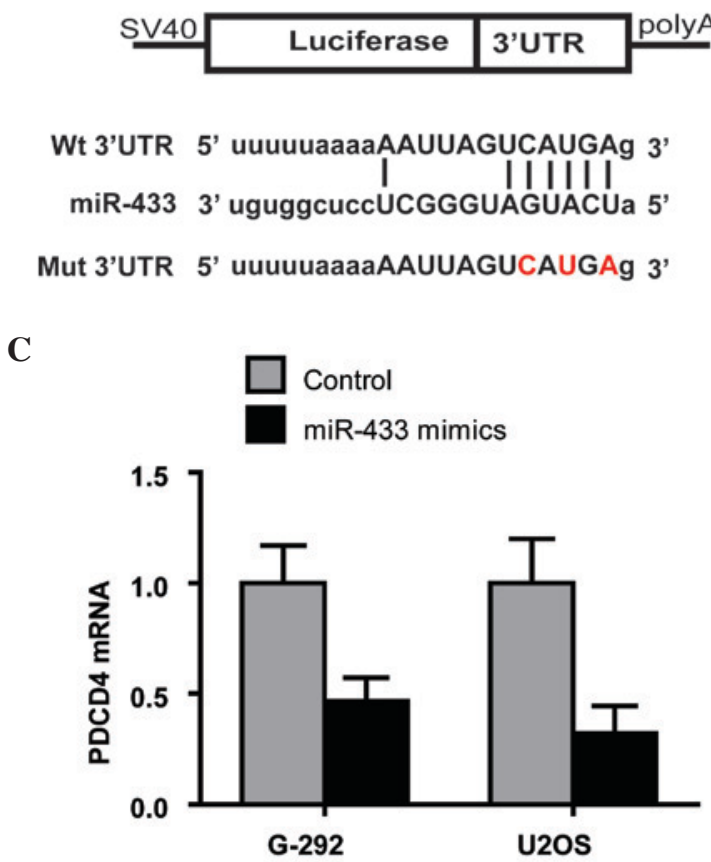

B

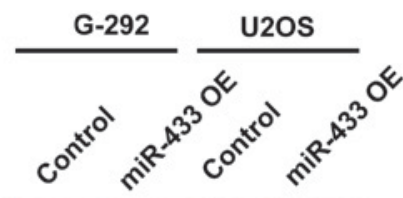

PDCD4

$\beta$-actin

D

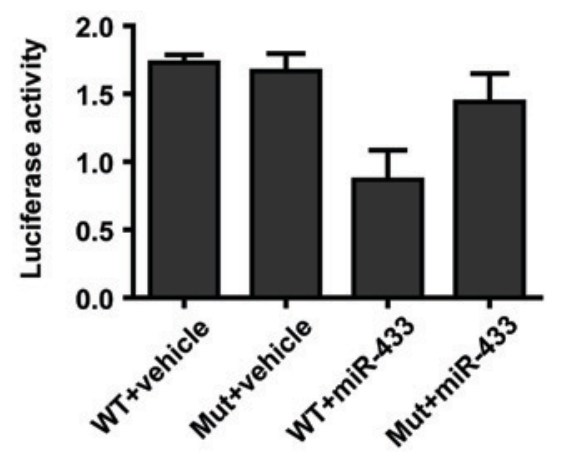

Figure 3. miR-433 decreases the expression of PDCD4 in osteosarcoma cells. (A) Sequences in the 3'UTR of PDCD4 mRNA were predicted to bind to miR-433. The red nucleotides were mutated to their complementary nucleotides. (B) Western blot analysis showed that overexpression of miR-433 inhibited the protein expression of PDCD4 in G-292 and U2OS cells. (C) Reverse transcription-quantitative polymerase chain reaction demonstrated that overexpression of miR-433 reduced the mRNA expression level of PDCD4 in G-292 and U2OS cells. (D) The luciferase reporter activity assay showed that miR-433 suppressed luciferase activity in the WT 3'UTR-transfected cells, but does not in the Mut 3'UTR-transfected cells. miR-433, microRNA-433; PDCD4, programmed cell death 4; 3'UTR, 3'-untranslated region; WT, wild type; Mut, mutant; OE, overexpression.

A

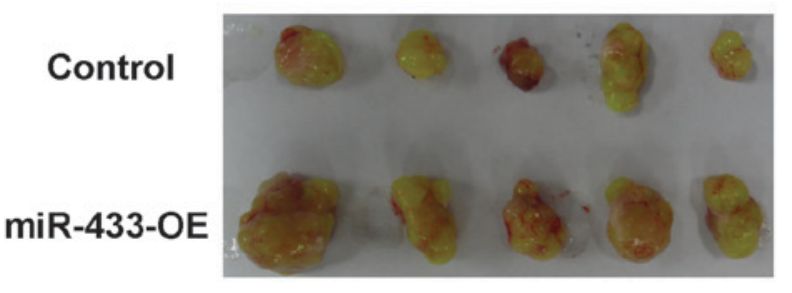

C

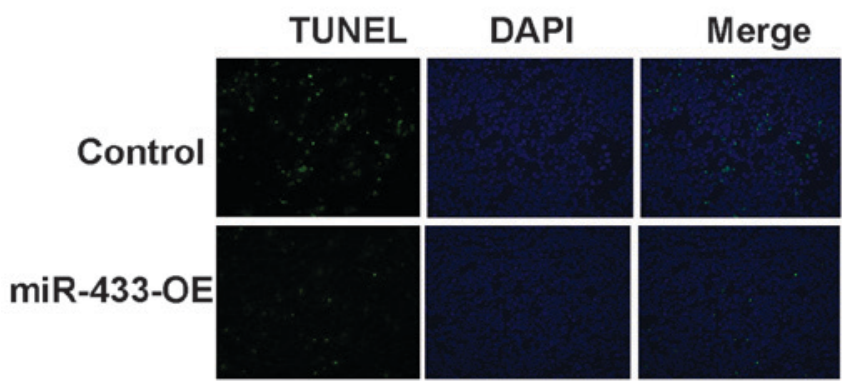

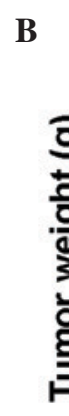

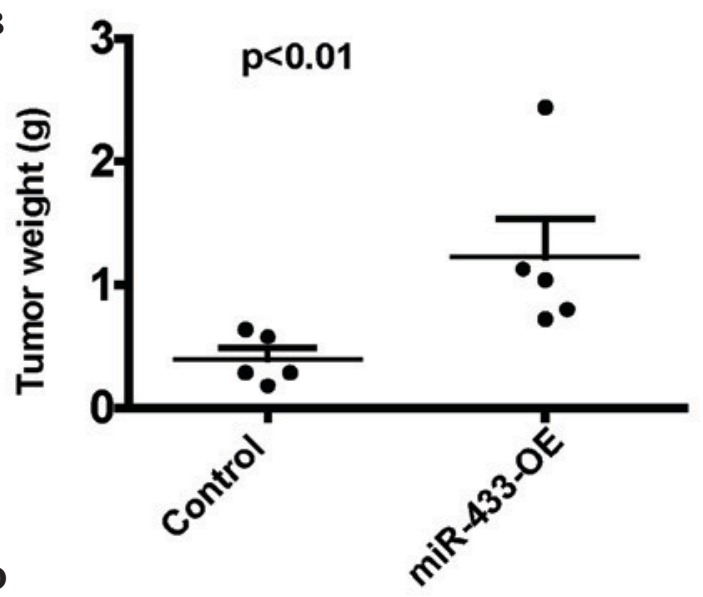

D

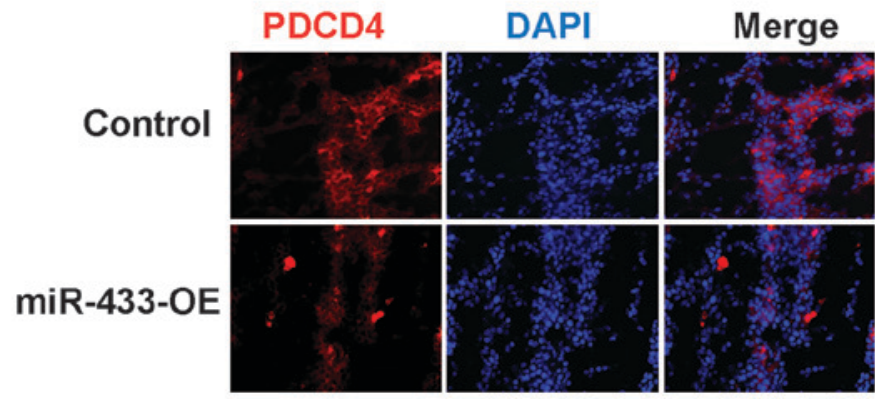

Figure 4. miR-433 decreases tumor cell apoptosis in vivo. (A) The photo is a representative image of the xenograft tumors. (B) Tumor weights were measured at 30 days post-injection in the two groups. (C) The TUNEL-positive cells were analyzed in the tumor sections (magnification, x20). (D) The expression of PDCD4 in xenograft tumors was analyzed by immunofluorescence. miR-433, microRNA-433; TUNEL, terminal deoxynucleotidyl transferase dUTP nick end labeling; PDCD4, programmed cell death 4; OE, overexpression. 
resistance in osteosarcoma (22-25). Some miRNAs function as oncogenes, and have been reported to be upregulated in osteosarcoma, while others serve as tumor suppressors, and are often downregulated in osteosarcoma (26). Therefore, a potential therapeutic strategy in osteosarcoma would be to knockdown the expression of oncogenic miRNAs using miRNA inhibitors or to overexpress the tumor suppressor miRNAs using miRNA mimics.

Previous reports have shown that miR-433 is aberrantly expressed in various cancers. For example, miR-433 was decreased in myeloproliferative neoplasms, and overexpression of miR-433 suppressed hematopoietic cell growth and differentiation by targeting GBP2 (10). The present study demonstrated that miR-433 was upregulated in osteosarcoma tissues compared with the adjacent normal tissues, and inhibited osteosarcoma apoptosis. The present data, along with an analysis of a published dataset (27), showed that miR-433 was overexpressed in osteosarcoma tissues and cell lines. In combination with previous reports revealing the roles of miR-433 in various other types of cancer, including ovarian and gastric cancer $(28,29)$, the present study further confirmed that miR-433 functions as an oncomiR in osteosarcoma. Furthermore, overexpression of miR-433 was shown to decrease the apoptosis of osteosarcoma cells by targeting PDCD4 in vitro. In addition, in the xenograft tumor model, miR-433-overexpressing U2OS cells were more resistant to apoptosis.

In conclusion, the present study is the first to demonstrate that miR-433 is significantly overexpressed in osteosarcoma tissues and cell lines. Furthermore, miR- 433 was shown to play a crucial role in apoptosis by targeting PDCD4 in osteosarcoma. These results suggest that miR-433 may be a molecular target for osteosarcoma therapy.

\section{References}

1. Ferguson WS and Goorin AM: Current treatment of osteosarcoma. Cancer Invest 19: 292-315, 2001.

2. Chou AJ and Gorlick R: Chemotherapy resistance in osteosarcoma: Current challenges and future directions. Expert Rev Anticancer Ther 6: 1075-1085, 2006.

3. Luetke A, Meyers PA, Lewis I and Juergens H: Osteosarcoma treatment-where do we stand? A state of the art review. Cancer Treat Rev 40: 523-532, 2014.

4. Aljubran AH, Griffin A, Pintilie M and Blackstein M: Osteosarcoma in adolescents and adults: Survival analysis with and without lung metastases. Ann Oncol 20: 1136-1141, 2009.

5. Ghosh T, Aprea J, Nardelli J, Engel H, Selinger C, Mombereau C, Lemonnier T, Moutkine I, Schwendimann L, Dori M, et al: MicroRNAs establish robustness and adaptability of a critical gene network to regulate progenitor fate decisions during cortical neurogenesis. Cell Rep 7: 1779-1788, 2014.

6. Mitra R, Sun J and Zhao Z: microRNA regulation in cancer: One arm or two arms? Int J Cancer 137: 1516-1518, 2015.

7. Treiber T, Treiber $\mathrm{N}$ and Meister G: Regulation of microRNA biogenesis and function. Thromb Haemost 107: 605-610, 2012.

8. Guo LH, Li H, Wang F, Yu J and He JS: The tumor suppressor roles of miR-433 and miR-127 in gastric cancer. Int J Mol Sci 14: 14171-14184, 2013.

9. Gotanda K, Hirota T, Matsumoto N and Ieiri I: MicroRNA-433 negatively regulates the expression of thymidylate synthase (TYMS) responsible for 5-fluorouracil sensitivity in HeLa cells. BMC Cancer 13: 369, 2013.
10. Lin X, Rice KL, Buzzai M, Hexner E, Costa FF, Kilpivaara O, Mullally A, Soares MB, Ebert BL, Levine R and Licht JD: miR-433 is aberrantly expressed in myeloproliferative neoplasms and suppresses hematopoietic cell growth and differentiation. Leukemia 27: 344-352, 2013.

11. Pfaffl MW: A new mathematical model for relative quantification in real-time RT-PCR. Nucleic Acids Res 29: e45, 2001.

12. Lin SM, Du P,Huber W and Kibbe WA: Model-based variance-stabilizing transformation for Illumina microarray data. Nucleic Acids Res 36: e11, 2008.

13. Luo F, Du X, Weng T, Wen X, Huang J and Chen L: Efficient multi-site-directed mutagenesis directly from genomic template. J Biosci 37: 965-969, 2012.

14. Lewis BP, Burge CB and Bartel DP: Conserved seed pairing, often flanked by adenosines, indicates that thousands of human genes are microRNA targets. Cell 120: 15-20, 2005.

15. Betel D, Wilson M, Gabow A, Marks DS and Sander C: The microRNA.org resource: targets and expression. Nucleic Acids Res 36: D149-D153, 2008.

16. Lankat-Buttgereit B and Göke R: The tumour suppressor Pdcd4: Recent advances in the elucidation of function and regulation. Biol Cell 101: 309-317, 2009.

17. Chen X, Bahrami A, Pappo A, Easton J, Dalton J, Hedlund E, Ellison D, Shurtleff S, Wu G, Wei L, et al: Recurrent somatic structural variations contribute to tumorigenesis in pediatric osteosarcoma. Cell Rep 7: 104-112, 2014.

18. Duong LM and Richardson LC: Descriptive epidemiology of malignant primary osteosarcoma using population-based registries, United States, 1999-2008. J Registry Manag 40: 59-64, 2013.

19. Zhao H, Ma B, Wang Y, Han T, Zheng L, Sun C, Liu T, Zhang Y, Qiu X and Fan Q: miR-34a inhibits the metastasis of osteosarcoma cells by repressing the expression of CD44. Oncol Rep 29: 1027-1036, 2013.

20. Wang Q, Cai J, Wang J, Xiong C and Zhao J: MiR-143 inhibits EGFR-signaling-dependent osteosarcoma invasion. Tumour Biol 35: 12743-12748, 2014.

21. Song B, Wang Y, Xi Y, Kudo K, Bruheim S, Botchkina GI, Gavin E, Wan Y, Formentini A, Kornmann M, et al: Mechanism of chemoresistance mediated by miR-140 in human osteosarcoma and colon cancer cells. Oncogene 28: 4065-4074, 2009.

22. Zhou C, Tan W, Lv H, Gao F and Sun J4: Hypoxia-inducible microRNA-488 regulates apoptosis by targeting Bim in osteosarcoma. Cell Oncol (Dordr) 39: 463-471, 2016.

23. Lin Z, Song D, Wei H, Yang X, Liu T, Yan W and Xiao J: TGF- $\beta 1$-induced miR-202 mediates drug resistance by inhibiting apoptosis in human osteosarcoma. J Cancer Res Clin Oncol 142: 239-246, 2016.

24. Wang GC, He QY, Tong DK, Wang CF, Liu K, Ding C, Ji F and Zhang H: MiR-367 negatively regulates apoptosis induced by adriamycin in osteosarcoma cells by targeting KLF4. J Bone Oncol 5: 51-56, 2016

25. Zhang Y, Duan G and Feng S: MicroRNA-301a modulates doxorubicin resistance in osteosarcoma cells by targeting AMP-activated protein kinase alpha 1. Biochem Biophys Res Commun 459: 367-373, 2015.

26. Ram Kumar RM, Boro A and Fuchs B: Involvement and clinical aspects of microRNA in osteosarcoma. Int J Mol Sci 17: E877, 2016.

27. Kelly AD, Haibe-Kains B, Janeway KA, Hill KE, Howe E, Goldsmith J, Kurek K, Perez-Atayde AR, Francoeur N, Fan JB, et al: MicroRNA paraffin-based studies in osteosarcoma reveal reproducible independent prognostic profiles at 14q32. Genome Med 5: 2, 2013.

28. Weiner-Gorzel K, Dempsey E, Milewska M, McGoldrick A, Toh V, Walsh A, Lindsay S, Gubbins L, Cannon A, Sharpe D et al: Overexpression of the microRNA miR-433 promotes resistance to paclitaxel through the induction of cellular senescence in ovarian cancer cells. Cancer Med 4: 745-758, 2015.

29. Guo LH, Li H, Wang F, Yu J and He JS: The tumor suppressor roles of mir-433 and mir-127 in gastric cancer. Int J Mol Sci 14: 14171-14184, 2013 